OPEN ACCESS

Edited and reviewed by:

Marcello Dallio,

University of Campania Luigi

Vanvitelli, Italy

*Correspondence:

Imke Atreya

imke.atreya@uk-erlangen.de

Specialty section:

This article was submitted to

Gastroenterology,

a section of the journal

Frontiers in Medicine

Received: 11 November 2021 Accepted: 25 November 2021 Published: 09 December 2021

Citation:

Weidinger C, Krug SM, Voskens C, Moschen AR and Atreya I (2021) Editorial: Loss of Epithelial Barrier Integrity in Inflammatory Diseases: Cellular Mediators and Therapeutic

Targets. Front. Med. 8:813153.

doi: 10.3389/fmed.2021.813153

\section{Editorial: Loss of Epithelial Barrier Integrity in Inflammatory Diseases: Cellular Mediators and Therapeutic Targets}

\author{
Carl Weidinger ${ }^{1}$, Susanne M. Krug ${ }^{2}$, Caroline Voskens ${ }^{3,4}$, Alexander R. Moschen ${ }^{5}$ and \\ Imke Atreya ${ }^{\text {6* }}$ \\ ${ }^{1}$ Division of Gastroenterology, Infectious Diseases and Rheumatology, Medical Department, Charité-Universitätsmedizin \\ Berlin, Berlin, Germany, ${ }^{2}$ Clinical Physiology/Nutritional Medicine, Charité-Universitätsmedizin Berlin, Berlin, Germany, \\ ${ }^{3}$ Department of Dermatology, University Hospital Erlangen, Friedrich-Alexander-Universität Erlangen-Nürnberg, Erlangen, \\ Germany, ${ }^{4}$ Deutsches Zentrum Immuntherapie (DZI), University Hospital Erlangen, Erlangen, Germany, ${ }^{5}$ Internal Medicine 2 \\ (Gastroenterology and Hepatology), Faculty of Medicine, Kepler University Hospital, Johannes Kepler University Linz, Linz, \\ Austria, ${ }^{6}$ Department of Medicine 1, University Hospital of Erlangen, Friedrich-Alexander-Universität Erlangen-Nürnberg, \\ Erlangen, Germany
}

Keywords: inflammatory bowel diseases, barrier integrity, immune-epithelial interactions, intestinal mucosa, IBD pathogenesis

\section{Editorial on the Research Topic}

Loss of Epithelial Barrier Integrity in Inflammatory Diseases: Cellular Mediators and Therapeutic Targets

\section{IMPAIRED INTESTINAL BARRIER INTEGRITY IN IBD}

About $0.75 \%$ of the inhabitants of the industrialized countries of the Western world were affected by one of the two main entities of inflammatory bowel diseases (IBD), Crohn's disease or ulcerative colitis, in 2020 (1). This means that a significant part of the general population is affected by chronically remitting gastroenterological symptoms, such as bloody diarrhea, abdominal pain, and anemia. Importantly, besides its high incidence, IBD mainly affects the younger population, who are often significantly hindered in their social and professional lives by the disease. Despite the successful clinical establishment of new hallmark therapies during the last three decades, about $40 \%$ of IBD patients do not satisfactorily respond to current treatment strategies or suffer a secondary loss of response (2). Together, this promotes a scientific and clinical interest in identifying innovative therapeutic targets (3), and in this course, there is a steadily growing improvement in knowledge regarding the exact molecular and cellular processes of IBD pathogenesis. Although in the past, many pioneering studies focused on intestinal immune cells (4-6), as their overwhelmingly enhanced activation status and cytokine release were long assumed as predominant drivers of IBD, nowadays, there is growing awareness that the entire mucosal barrier and its function decisively determines the development and resolution of chronic intestinal inflammation (7-9). Addressing this aspect in our Research Topic, we present a diverse collection of original and review articles focusing on the pathological dysregulation and clinical relevance of the mucosal barrier integrity and its cellular key players in IBD.

Besides absorptive and secretory intestinal epithelial cells (IECs) forming the tightly closed epithelial monolayer, a broad spectrum of locally accumulating immune cells including also innate 
lymphoid cells as a rather newly described immune cell population (reviewed by Schulz-Kuhnt et al.), as well as nonhematopoietic cells, such as endothelial cells, enteric neurons, adipocytes, and fibroblasts, contribute to the protection of the intestinal lamina propria against potentially invading luminal pathogens or foreign antigens (9-16). Furthermore, numerous intestinal blood vessels are located in direct proximity to the epithelial layer and are influenced by local inflammatory processes (e.g., IFN $\gamma$-induced disruption of endothelial barrier integrity) (17), while, vice versa, the inflammation-triggered increased blood flow supports recruitment of pro-inflammatory immune cells from the circulation (comprehensively summarized by Stürzl et al.). In addition, the enteric nervous system represents another factor of impact on intestinal homeostasis that has been neglected in the past and whose potential contribution in intestinal inflammation and impaired gut barrier is discussed by Drobny et al. While we are constantly getting better understanding of how the dysregulated function of individual cell populations in the gut contributes to the loss of intestinal homeostasis and the initiation or maintenance of chronic inflammatory processes, it is also becoming increasingly clear that the real challenge is deciphering the communication between the different components of this cellular network and to identify central molecular switches driving the loss of mucosal barrier integrity, as well as the counteracting process of mucosal wound healing in IBD (summarized by Sommer et al.). For sure, immune cell-derived cytokines represent central signaling molecules within this and a study by Delbue et al. provides new mechanistic insights into IL-22-mediated effects on epithelial integrity and wound healing. Moreover, implying even higher complexity, the interplay between different cellular compartments of the gut may further be influenced by external factors derived from the lumen, as very well-established for intestinal microbiota (an overview is provided by Jergens et al.) and of clear relevance also for defined nutritional components $(18,19)$. For example, Yeung et al. observed that reduced uptake of vitamin D resulted in impaired mucosal barrier properties.

\section{IMPLICATIONS FOR IBD THERAPY}

Most of the clinically applied strategies in IBD therapy, including classic immunosuppressive drugs (e.g., azathioprine and 6-mercaptopurine), but also more specific approaches like anti-cytokine antibodies (e.g., anti-TNF therapy and IL12/IL-23-neutralizing ustekinumab) and anti-adhesion therapy (e.g., vedolizumab) primarily target the pathologically increased activation and/or accumulation of pro-inflammatory immune cells in the intestinal mucosa $(3,6)$. Lately, the maintenance

\section{REFERENCES}

1. Kaplan GG, Windsor JW. The four epidemiological stages in the global evolution of inflammatory bowel disease. Nat Rev Gastroenterol Hepatol. (2021) 18:56-66. doi: 10.1038/s41575-020-00360-x

2. Atreya R, Neurath MF, Siegmund B. Personalizing treatment in ibd: hype or reality in 2020? Can we predict response to and restoration of the intestinal barrier function and mucosal healing emerged as relatively new therapeutic goal in the clinical management of $\operatorname{IBD}(8,20)$ and this also resulted in a better consideration of the epithelial-protective effects of established therapeutics. For example, the recognized capacity of anti-TNF therapy to restore the pathologically increased rate of apoptotic IECs and the subsequent loss of epithelial resistance in IBD patients (21), as well as the counteracting influence of azathioprine and 5-aminosalicylic acid on the inflammation-triggered downregulation and rearrangement of junctional proteins in in vitro cultured IECs and intestinal organoids (22). In addition, defined molecular mediators and intracellular signaling pathways involved in the maintenance of the epithelial tightness (e.g., STAT6, angulin-1, leptin, RhoA, and IL13R $\alpha 2$ ) (23-26), in IEC survival (e.g., Caspase-8) (27), in the production of antimicrobial peptides (e.g., human $\beta$-defensin 2 ) (28) and in wound healing (e.g., STAT1, STAT3) $(29,30)$ have also been suggested as innovative therapeutic targets. As exemplarily demonstrated by Gerbeth et al. summarizing the multiple effects of histone deacetylase inhibitors on gut homeostasis, it will in general be essential to always consider the above outlined cellular complexity of the protective mucosal barrier and carefully validate the role of potential innovative target structures for the entire panel of involved cell types. Moreover, the fact that pathological conditions significantly differ dependent on the phase of disease (nicely described by Semin et al.) and its site of manifestation (emphasized by the study of Stolzer et al. describing a different impact of STAT1 signaling on IEC cell death in the context of inflammation in ileum and colon, and by comparative transcriptomic results reported by Gonzalez Acera et al.), makes it important to also develop diagnostic strategies allowing a careful clinical and molecular characterization of the individual disease status prior to the selection and initiation of therapy. In this context, Bojarski et al. provide a valuable overview of innovative advanced gastrointestinal endoscopic technologies and their potential future contribution in paving the way for personalized medicine in IBD.

\section{AUTHOR CONTRIBUTIONS}

All authors listed have made a substantial, direct, and intellectual contribution to the work and approved it for publication.

\section{FUNDING}

This work has received funding from the DFG, German Research Foundation (SFB TransRegio TRR 241, subprojects A07, B01, $\mathrm{B} 06$, and C04). 
mice reconstituted with CD45RBhi CD4+ T cells. Immunity. (1994) 1:553-62. doi: 10.1016/1074-7613(94)90045-0

5. Fuss IJ, Neurath M, Boirivant M, Klein JS, de la Motte C, Strong SA, et al. Disparate CD4+ lamina propria (LP) lymphokine secretion profiles in inflammatory bowel disease. Crohn's disease LP cells manifest increased secretion of IFN-gamma, whereas ulcerative colitis LP cells manifest increased secretion of IL-5. J Immunol. (1996) 157:1261-70. doi: 10.1097/00024382-199703001-00528

6. Gerner RR, Moschen AR, Tilg H. Targeting $\mathrm{T}$ and $\mathrm{B}$ lymphocytes in inflammatory bowel diseases: lessons from clinical trials. Digest Dis. (2013) 31:328-35. doi: 10.1159/000354687

7. Stange EF, Schroeder BO. Microbiota and mucosal defense in IBD: an update. Exp Rev Gastroenterol Hepatol. (2019) 13:963-76. doi: $10.1080 / 17474124.2019 .1671822$

8. Schoultz I, Keita AV. Cellular and molecular therapeutic targets in inflammatory bowel disease-focusing on intestinal barrier function. Cells. (2019) 8:193. doi: 10.3390/cells8020193

9. Martini E, Krug SM, Siegmund B, Neurath MF, Becker C. Mend your fences: the epithelial barrier and its relationship with mucosal immunity in inflammatory bowel disease. Cell Mol Gastroenterol Hepatol. (2017) 4:33-46. doi: 10.1016/j.jcmgh.2017.03.007

10. Lopez-Posadas R, Neurath MF, Atreya I. Molecular pathways driving diseasespecific alterations of intestinal epithelial cells. Cell Mol Life Sci. (2017) 74:803-26. doi: 10.1007/s00018-016-2363-2

11. Scheibe K, Backert I, Wirtz S, Hueber A, Schett G, Vieth M, et al. IL-36R signalling activates intestinal epithelial cells and fibroblasts and promotes mucosal healing in vivo. Gut. (2017) 66:823-38. doi: 10.1136/gutjnl-2015-310374

12. Weidinger C, Hegazy AN, Siegmund B. The role of adipose tissue in inflammatory bowel diseases. Curr Opin Gastroenterol. (2018) 34:183-6. doi: 10.1097/MOG.0000000000000445

13. Brun P, Giron MC, Qesari M, Porzionato A, Caputi V, Zoppellaro C, et al. Toll-like receptor 2 regulates intestinal inflammation by controlling integrity of the enteric nervous system. Gastroenterology. (2013) 145:1323-33. doi: 10.1053/j.gastro.2013.08.047

14. Danese S, Fiocchi C. Endothelial cell-immune cell interaction in IBD. Digest Dis. (2016) 34:43-50. doi: 10.1159/000442925

15. Sans M, Danese S, de la Motte C, de Souza HS, Rivera-Reyes BM, West GA, et al. Enhanced recruitment of CX3CR1 + T cells by mucosal endothelial cellderived fractalkine in inflammatory bowel disease. Gastroenterology. (2007) 132:139-53. doi: 10.1053/j.gastro.2006.10.010

16. Tilg $\mathrm{H}$, Moschen AR. Adipocytokines: mediators linking adipose tissue, inflammation and immunity. Nat Rev Immunol. (2006) 6:772-83. doi: 10.1038/nri1937

17. Haep L, Britzen-Laurent N, Weber TG, Naschberger E, Schaefer A, Kremmer $\mathrm{E}$, et al. Interferon gamma counteracts the angiogenic switch and induces vascular permeability in dextran sulfate sodium colitis in mice. Inflamm Bowel Dis. (2015) 21:2360-71. doi: 10.1097/MIB.0000000000000490

18. Mayr L, Grabherr F, Schwarzler J, Reitmeier I, Sommer F, Gehmacher T, et al. Dietary lipids fuel GPX4-restricted enteritis resembling Crohn's disease. Nat Commun. (2020) 11:1775. doi: 10.1038/s41467-020-15646-6

19. Caenepeel C, Sadat Seyed Tabib N, Vieira-Silva S, Vermeire S. Review article: how the intestinal microbiota may reflect disease activity and influence therapeutic outcome in inflammatory bowel disease. Aliment Pharmacol Ther. (2020) 52:1453-68. doi: 10.1111/apt.16096

20. Liu CY, Cham CM, Chang EB. Epithelial wound healing in inflammatory bowel diseases: the next therapeutic frontier. Transl Res. (2021) 236:35-51. doi: $10.1016 /$ j.trsl.2021.06.001
21. Zeissig S, Bojarski C, Buergel N, Mankertz J, Zeitz M, Fromm M, et al. Downregulation of epithelial apoptosis and barrier repair in active Crohn's disease by tumour necrosis factor alpha antibody treatment. Gut. (2004) 53:1295-302. doi: 10.1136/gut.2003.03 6632

22. Khare V, Krnjic A, Frick A, Gmainer C, Asboth M, Jimenez $\mathrm{K}$, et al. Mesalamine and azathioprine modulate junctional complexes and restore epithelial barrier function in intestinal inflammation. Sci Rep. (2019) 9:2842. doi: 10.1038/s41598-019-39 401-0

23. Lin $\mathrm{Y}$, Li B, Yang $\mathrm{X}$, Liu $\mathrm{T}$, Shi $\mathrm{T}$, Deng $\mathrm{B}$, et al. Non-hematopoietic STAT6 induces epithelial tight junction dysfunction and promotes intestinal inflammation and tumorigenesis. Mucosal Immunol. (2019) 12:1304-15. doi: 10.1038/s41385-019-0204-y

24. Hu JE, Bojarski C, Branchi F, Fromm M, Krug SM. Leptin downregulates angulin-1 in active crohn's disease via STAT3. Int J Mol Sci. (2020) 21:7824. doi: 10.3390/ijms 21217824

25. Krug SM, Bojarski C, Fromm A, Lee IM, Dames P, Richter JF, et al. Tricellulin is regulated via interleukin-13-receptor alpha2, affects macromolecule uptake, and is decreased in ulcerative colitis. Mucosal Immunol. (2018) 11:345-56. doi: $10.1038 / \mathrm{mi} .2017 .52$

26. Lopez-Posadas R, Becker C, Gunther C, Tenzer S, Amann K, Billmeier U, et al. Rho-A prenylation and signaling link epithelial homeostasis to intestinal inflammation. J Clin Invest. (2016) 126:611-26. doi: 10.1172/JCI80997

27. Gunther C, Martini E, Wittkopf N, Amann K, Weigmann B, Neumann H, et al. Caspase-8 regulates TNF-alpha-induced epithelial necroptosis and terminal ileitis. Nature. (2011) 477:335-9. doi: 10.1038/nature10400

28. Koeninger L, Armbruster NS, Brinch KS, Kjaerulf S, Andersen B, Langnau C, et al. Human beta-Defensin 2 mediated immune modulation as treatment for experimental colitis. Front Immunol. (2020) 11:93. doi: 10.3389/fimmu.2020.00093

29. Chiriac MT, Buchen B, Wandersee A, Hundorfean G, Gunther C, Bourjau Y, et al. Activation of epithelial signal transducer and activator of transcription 1 by interleukin 28 controls mucosal healing in mice with colitis and is increased in mucosa of patients with inflammatory bowel disease. Gastroenterology. (2017) 153:123-38.e8. doi: 10.1053/j.gastro.2017.03.015

30. Pickert G, Neufert C, Leppkes M, Zheng Y, Wittkopf N, Warntjen M, et al. STAT3 links IL-22 signaling in intestinal epithelial cells to mucosal wound healing. J Exp Med. (2009) 206:1465-72. doi: 10.1084/jem.20082683

Conflict of Interest: The authors declare that the research was conducted in the absence of any commercial or financial relationships that could be construed as a potential conflict of interest.

Publisher's Note: All claims expressed in this article are solely those of the authors and do not necessarily represent those of their affiliated organizations, or those of the publisher, the editors and the reviewers. Any product that may be evaluated in this article, or claim that may be made by its manufacturer, is not guaranteed or endorsed by the publisher.

Copyright $\odot 2021$ Weidinger, Krug, Voskens, Moschen and Atreya. This is an openaccess article distributed under the terms of the Creative Commons Attribution License (CC BY). The use, distribution or reproduction in other forums is permitted, provided the original author(s) and the copyright owner(s) are credited and that the original publication in this journal is cited, in accordance with accepted academic practice. No use, distribution or reproduction is permitted which does not comply with these terms. 